\title{
A unified criterion for the growth and coalescence of microvoids
}

\author{
Léo Morin $^{\mathrm{a}, *}$, Jean-Baptiste Leblond ${ }^{\mathrm{a}}$, A. Amine Benzerga ${ }^{\mathrm{b}, \mathrm{c}}$, \\ Djimédo Kondo ${ }^{\text {a }}$ \\ ${ }^{a}$ Sorbonne Universités, UPMC Univ Paris 06, CNRS, UMR 7190, Institut Jean Le Rond \\ d'Alembert, F-75005 Paris, France \\ ${ }^{\mathrm{b}}$ Department of Aerospace Engineering, Texas A\&M University, College Station, TX \\ 77843-3141, USA \\ ${ }^{\mathrm{c}}$ Department of Materials Science $\mathcal{E}$ Engineering, Texas A $6 M$ University, College Station, TX \\ 77843-3141, USA
}

\begin{abstract}
A yield criterion is developed which unifies void growth and void coalescence theories. Standard void growth theory assumes that plastic flow is diffuse, if not prevalent everywhere within the matrix of the elementary cell considered. On the other hand, void coalescence theory assumes states of post-localized plasticity whereby plastic flow is restricted to intervoid ligaments. The new theory accommodates both scenarios through some appropriate choice of microscopic velocity fields. An important implication for actual evolution problems is a seamless transition from void growth to void coalescence. This is in contrast with previous hybrid approaches whereby abrupt transitions are associated with the presence of unavoidable corners in the effective yield surface. More generally, the new criterion is applicable to describe yielding in porous metal plasticity for both low and high void volume fractions.

Keywords: Porous metal plasticity; Growth of voids; Coalescence of voids; Limit-analysis; Finite element simulations
\end{abstract}

\section{Introduction}

Early models of failure in ductile metals were based on analyses of the growth of isolated voids in infinite plastic matrices (McClintock, 1968; Rice and Tracey, 1969; Cocks and Ashby, 1982). In such situations the porosity (void volume fraction) is zero. In order to account for non-zero porosities, later studies considered finite, supposedly "representative" elementary cells containing one or several voids. Such micromechanical analyses enable scale transitions whereby the macroscopic response explicitly depends on a set of

* Corresponding author.

Email address: leo.morin@ens-cachan.fr (Léo Morin). 
microstructural parameters related to the voids and their arrangement (Ponte Castaneda and Suquet, 1997; Benzerga and Leblond, 2010). In these formulations, damage or void growth rate equations are a natural outcome.

The focus of the present work is on void growth (for weakly interacting voids) and coalescence (for strongly interacting voids) in rate-independent, plastic solids (at room or moderate temperatures). For such materials the theory of limit analysis (Prager and Hodge, 1951; Suquet, 1983), which amounts to idealizing the material behavior as rigid-ideal plastic (extreme case of nonlinearity), represents a natural framework of analysis.

The applications in view essentially pertain to the ductile failure of metals. In such a case the microstructure continuously evolves, but the porosities reached remain moderate. It is worth noting, however, that a modeling framework for void growth and coalescence should also permit to consider the very different case of cellular materials, for which the microstructure remains fixed but the porosity may be very high (Deshpande and Fleck, 2000). Most existing models of porous metal plasticity are truly applicable in the sole regime of low porosity (typically below 0.01), and are therefore not fit to such materials; but a combined void growth/coalescence model must necessarily consider higher porosities.

Since the seminal work of Gurson (1977), the modeling of void growth in plastic solids with finite porosity has known major improvements, especially during the past two decades or so (see notably Gologanu et al. (1993, 1994, 1997); Benzerga and Besson (2001); Monchiet et al. (2008); Keralavarma and Benzerga (2010); Madou and Leblond (2012a,b, 2013); Madou et al. (2013); Morin et al. (2015b)). On the other hand, the modeling of void coalescence is still in its infancy, because of the major difficulties arising from the large interactions between voids implied by this phenomenon. The reader is referred to the recent papers of Morin et al. (2015a) and Torki et al. (2015) for a recollection of developments in the field. Some of the difficulties met are as follows:

- Unsuccessful attempts have been made to interpret coalescence as a phenomenon of strain localization, at the scale of some homogenized model incorporating the presence of voids. Appendix A provides a brief description of these attempts and comments on their failure.

- Usual homogenization theories do not apply either to coalescence. Indeed such theories rely on an assumption of clear separation of scales which is generally not satisfied in the case of coalescence of voids, as detailed in Appendix B.

As a consequence, the seemingly most promising approaches of coalescence rely on analyses of elementary mechanisms but do not, strictly speaking, pertain to homogenization because they consider cells of fixed, small dimensions, containing very few voids.

The first theoretical formulation of coalescence may be traced back to (Thomason, 1985), although a full treatment of the problem considered was provided only recently by (Benzerga and Leblond, 2014). These works considered simple geometries and loadings. Benzerga and Leblond (2014) developed a closed-form macroscopic yield criterion for a porous material in which the voids are in a "state of coalescence". By this expression is meant a state where plastic flow is entirely confined to horizontal intervoid ligaments, the regions 
above and below the voids being prevented from any deformation. Benzerga and Leblond (2014) considered a cylindrical cell containing a truncated cylindrical void, and subjected to boundary conditions allowing for strain localization within the intervoid ligament, that is imposing a vanishing lateral overall strain rate. In doing so, they used a set of kinematically admissible velocity fields resembling those used by Gurson, but only in the ligaments. Because of their simplicity, these velocity fields were discontinuous within the elementary cell. While such fields are unphysical, they are kinematically admissible and lead to a rigorous upper bound for the yield surface. As a matter of fact, finite-elementbased limit-analysis calculations performed by Morin et al. (2015a) have shown that the upper bound obtained by Benzerga and Leblond (2014) is quite good for a broad range of microstructural parameters.

In addition, Morin et al. (2015a) developed improved analytical models for the problem considered by Benzerga and Leblond (2014). They employed two new sets of velocity fields: a family of generalized discontinuous fields which considered the shape of the plastic/rigid interface as an adjustable parameter; and continuous velocity fields. The improved models provide, in general, tighter upper bounds than Benzerga and Leblond (2014)'s model, albeit for different ranges of structural parameters. Even more recently, Torki et al. (2015) extended Benzerga and Leblond (2014)'s model by considering combined shear and triaxial tension. Their model may be viewed as an elaboration of an earlier model by Tekoglu et al. (2012) who did not explicitly specify the microscopic velocity fields responsible for void expansion.

When boundary conditions enforcing zero lateral straining are not imposed a priori, the question arises as to what the effective yield state is. A point of particular importance here is that when the problem is solved computationally, with no recourse to approximate trial velocity fields, the exact yield locus of the porous solid under axisymmetric loadings exhibits both curved and flat parts (Morin et al., 2015a). These distinct parts correspond to loadings that are supported with plastic flow either filling the matrix or localized in the intervoid ligaments, respectively; the orientation of the flat part is dictated, via the normality property, by the peculiar direction of plastic flow imposed by the coalescence process (corresponding to a uniaxial extension). This feature of the yield locus is disregarded by Gurson-like models because they tacitly assume uniform boundary conditions for all stress and microstructural states. This type of yield locus is not either what the recent coalescence models of (Benzerga and Leblond, 2014; Morin et al., 2015a) predict because these pertain exclusively to coalescence states, having a zero lateral strain rate. Therefore, the objective of the present work is to develop expressions for the macroscopic (or effective) yield locus that mimic essential features of the exact one obtained numerically by Morin et al. (2015a). Such yield loci are said to achieve a unified description of void growth and coalescence.

To place the present effort in perspective, it is worth mentioning that previous works on void growth and coalescence have essentially dealt with the two phenomena separately (Zhang and Niemi, 1994; Benzerga et al., 1999; Pardoen and Hutchinson, 2000; Benzerga, 2002). As a consequence, the effective domain of reversibility was eventually viewed as the intersection of two approximate reversibility domains obtained independently, so that the yield surface (boundary of the reversibility domain) inevitably exhibited some corners; see 
e.g. Fig. 5 in (Benzerga, 2002). While this kind of "hybrid" approach has been successfully used to model fracture in 3D initially crack-free specimens (Benzerga et al., 2004), the coincidence of the corner on the yield surface with the first instance of coalescence may trigger plastic flow localization at the macroscopic scale (Benzerga et al., 2002); and it is not clear whether such a localization is "real" or simply arises from the approximations made in the constitutive model. The development of a unified framework of void growth and coalescence should permit to settle interrogations of this kind.

\section{Preliminaries}

\subsection{Geometry, material, admissibility conditions of velocity fields}

To define the overall behavior of the porous medium, a cylindrical "elementary cell" $\Omega$ containing a coaxial cylindrical void $\omega$ is considered. The void is truncated by horizontal planes, as depicted in Fig. 1 on a quadrant of the cell. Following previous work (Thomason, 1985; Benzerga and Leblond, 2014; Morin et al., 2015a) non-symmetric deformation modes, if any, are excluded in the present analysis. (Coalescence between voids which are not first neighbors, for instance, could generate such modes; but this is not a likely occurrence). Although this cell cannot be duplicated and stacked up to build a periodic lattice, it is a reasonable approximation of more complex geometries, such as hexagonal-prismatic or square-prismatic ones, rigorously defining a unit cell in a periodic lattice.

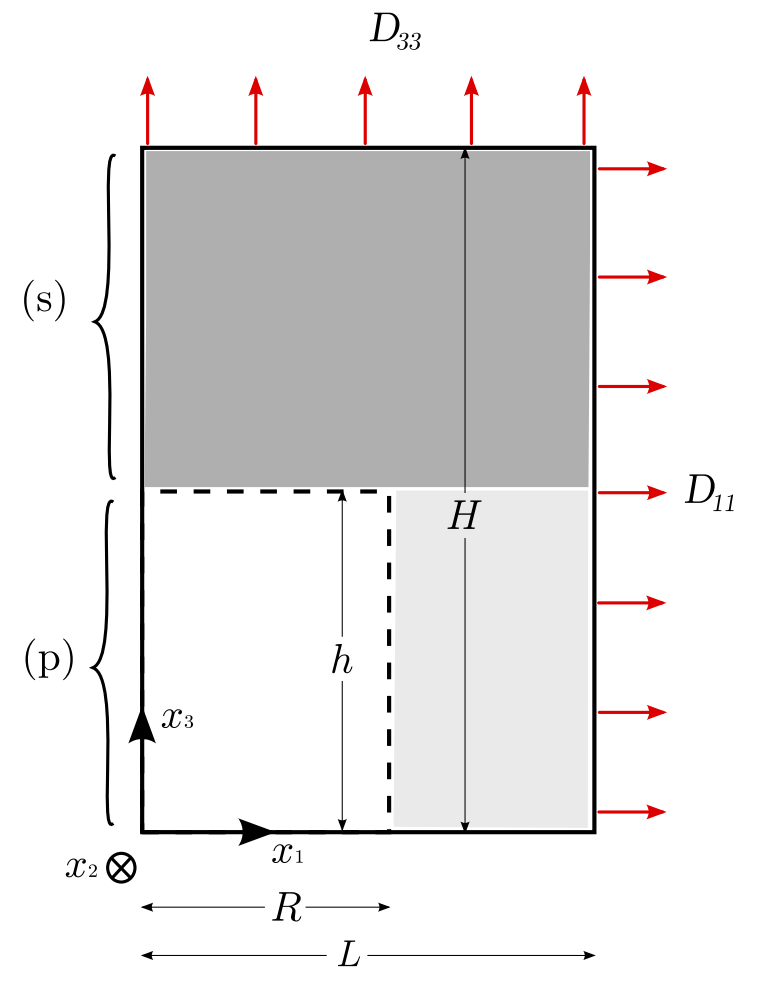

Fig. 1. Definition of the geometric parameters of the cylindrical elementary cell

The cylindrical geometry is characterized by the void aspect ratio $W=h / R$, the ligament 
parameter $\chi=R / L$ and the cell aspect ratio $\lambda=H / L$ (see Fig. 1). A fourth, useful dimensionless parameter related to the first three is the volume fraction of the voided band $c=h / H=W \chi / \lambda$. The local orthonormal basis associated to the cylindrical coordinates $r, \theta, z$ is denoted $\left(\mathbf{e}_{r}, \mathbf{e}_{\theta}, \mathbf{e}_{z}\right)$, and that associated to the Cartesian coordinates $x_{1}, x_{2}, x_{3}$ is denoted $\left(\mathbf{e}_{1}, \mathbf{e}_{2}, \mathbf{e}_{3}\right)$, with $\mathbf{e}_{3}=\mathbf{e}_{z}$.

The matrix is assumed to be rigid-ideal-plastic and obey the von Mises criterion:

$$
f(\boldsymbol{\sigma})=\sigma_{\text {eq }}^{2}-\sigma_{0}^{2} \leq 0 \quad \forall \mathbf{x} \in \Omega-\omega
$$

where $\sigma_{\text {eq }}$ is the von Mises equivalent stress and $\sigma_{0}$ the yield stress in simple tension, and the Prandtl-Reuss flow rule associated to this criterion via the normality property.

The cylindrical cell is subjected to boundary conditions of the following form:

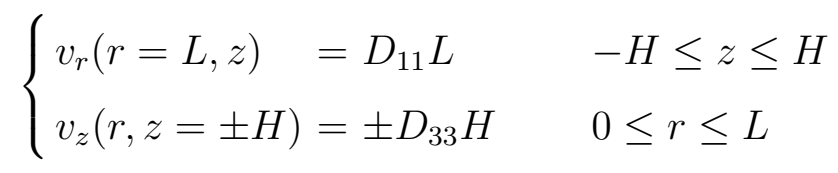

where $\mathbf{v}$ denotes the velocity, the orthoradial component $v_{\theta}$ being zero everywhere because of axial symmetry. Such boundary conditions, adapted to the cylindrical geometry, mimic periodic ones on a cubical cell. (The choice of a cylindrical cell, dictated by considerations of simplicity, prohibits to consider truly periodic boundary conditions).

In the plastic matrix, the velocity must verify the property of incompressibility imposed by the flow rule:

$$
\operatorname{tr} \mathbf{d}=\operatorname{div} \mathbf{v}=0 \quad, \quad \forall \mathbf{x} \in \Omega-\omega
$$

\subsection{Principles of the limit-analysis}

The classical inequality of limit-analysis reads:

$$
\Sigma: \mathbf{D} \leq \Pi(\mathbf{D}) \quad, \quad \forall \mathbf{D}
$$

where $\boldsymbol{\Sigma}$ and $\mathbf{D}$ are the macroscopic stress and strain rate tensors, defined as the volume averages of their microscopic counterparts $\boldsymbol{\sigma}$ and $\mathbf{d}$, and $\Pi(\mathbf{D})$ the macroscopic plastic dissipation. This dissipation is defined, in the case of a von Mises material, by the formula

$$
\Pi(\mathbf{D})=\inf _{\mathbf{v} \in \mathcal{K}(\mathbf{D})}\left\langle\sigma_{0} d_{\mathrm{eq}}\right\rangle_{\Omega}
$$

where $\mathcal{K}(\mathbf{D})$ is the set of velocity fields $\mathbf{v}$ verifying the properties mentioned in Subsection 2.1 for the given value of $\mathbf{D}$, and $d_{\text {eq }}$ the von Mises equivalent strain rate associated to the velocity field $\mathbf{v}$.

Because of the symmetries of the problem, the left-hand side in equation (4) reduces to

$$
\begin{aligned}
\boldsymbol{\Sigma}: \mathbf{D} & =2 \Sigma_{11} D_{11}+\Sigma_{33} D_{33} \\
& =3 \Sigma_{11} D_{\mathrm{m}}+\left(\Sigma_{33}-\Sigma_{11}\right) D_{33}
\end{aligned}
$$


where $D_{\mathrm{m}}=\frac{1}{3} \operatorname{tr} \mathbf{D}=\frac{1}{3}\left(2 D_{11}+D_{33}\right)$ is the mean strain rate. This suggests to define the following load parameters:

$$
\left\{\begin{array}{l}
\bar{\Sigma}_{1}=\Sigma_{11} \\
\bar{\Sigma}_{2}=\Sigma_{33}-\Sigma_{11}
\end{array}\right.
$$

together with the "conjugate" kinematic parameters

$$
\left\{\begin{array}{l}
\bar{D}_{1}=3 D_{\mathrm{m}} \\
\bar{D}_{2}=D_{33} .
\end{array}\right.
$$

(It may be noted that a seemingly more natural choice of conjugate parameters would consist of using $\Sigma_{\mathrm{m}}=\frac{1}{3} \operatorname{tr} \Sigma=\frac{1}{3}\left(2 \Sigma_{11}+\Sigma_{33}\right)$ and $\Sigma_{33}-\Sigma_{11}$ for the stresses, versus $3 D_{\mathrm{m}}$ and $\frac{2}{3}\left(D_{33}-D_{11}\right)$ for the strain rates; but the choice made in equations (7) and (8) leads to simpler calculations and expressions for the cylindrical geometry considered). With these variables, equation (4) reads

$$
\bar{\Sigma}_{1} \bar{D}_{1}+\bar{\Sigma}_{2} \bar{D}_{2} \leq \Pi\left(\bar{D}_{1}, \bar{D}_{2}\right) \quad, \quad \forall\left(\bar{D}_{1}, \bar{D}_{2}\right) .
$$

The macroscopic reversibility domain of the porous material, in the 2D space of macroscopic stresses $\left(\bar{\Sigma}_{1}, \bar{\Sigma}_{2}\right)$, is the intersection of the half-planes defined by these inequalities, $\bar{D}_{1}$ and $\bar{D}_{2}$ acting as parameters. The boundary of this domain, that is the macroscopic yield locus of the porous material, is the envelope of the straight lines $\bar{\Sigma}_{1} \bar{D}_{1}+\bar{\Sigma}_{2} \bar{D}_{2}=$ $\Pi\left(\bar{D}_{1}, \bar{D}_{2}\right), \bar{D}_{1}$ and $\bar{D}_{2}$ acting again as parameters. The regular part of this envelope is defined by the parametric equations

$$
\left\{\begin{array}{l}
\bar{\Sigma}_{1}=\frac{\partial \Pi}{\partial \bar{D}_{1}}\left(\bar{D}_{1}, \bar{D}_{2}\right) \\
\bar{\Sigma}_{2}=\frac{\partial \Pi}{\partial \bar{D}_{2}}\left(\bar{D}_{1}, \bar{D}_{2}\right) .
\end{array}\right.
$$

\section{Trial velocity fields}

The trial velocity field looked for must describe both the growth and coalescence stages. The growth phase corresponds to diffuse plasticity in the entire cell. On the other hand, the coalescence phase is characterized by a sudden concentration of the strain rate within the horizontal ligament connecting neighboring voids, implying the presence of rigid regions above and below the void.

To account for this concentration, Benzerga and Leblond (2014) proposed to divide the cell into two parts, a porous one $\Omega^{(\mathrm{p})}$ and a sound one $\Omega^{(\mathrm{s})}$ (see Fig.1). The velocity field they proposed to describe coalescence consisted of: (i) a radial expansion combined with a uniform axial extension of the porous zone, satisfying the condition of vanishing horizontal overall strain rate $D_{11}=0$ (special case of Gurson (1977)'s "cylindrical velocity field"); (ii) rigid-body motions of the sound regions. In order to also describe the growth of the 
void prior to localization of the strain rate within the porous zone, we shall extend this field by (i) dropping the condition $D_{11}=0$ - the velocity field in the porous zone then becomes identical to Gurson (1977)'s general "cylindrical velocity field"; (ii) allowing the sound regions to deform uniformly.

In order to benefit from Gurson (1977)'s classical solution for cylindrical voids, it will be useful to consider the overall "partial strain rates" $\mathbf{D}^{(\mathrm{p})}, \mathbf{D}^{(\mathrm{s})}$ in the porous and sound regions, respectively. The axial and horizontal components of these strain rates verify the obvious relations

$$
\left\{\begin{array}{l}
D_{33}=c D_{33}^{(\mathrm{p})}+(1-c) D_{33}^{(\mathrm{s})} \\
D_{11}=D_{11}^{(\mathrm{p})}=D_{11}^{(\mathrm{s})}
\end{array}\right.
$$

In addition, incompressibility of the sound zones implies that

$$
D_{33}^{(\mathrm{s})}=-2 D_{11}
$$

Combination of these relations implies that

$$
\left\{\begin{array}{l}
D_{33}^{(\mathrm{p})}=\frac{1}{c}\left[3(1-c) D_{\mathrm{m}}+c D_{33}\right] \\
D_{\mathrm{m}}^{(\mathrm{p})}=\frac{D_{\mathrm{m}}}{c}
\end{array}\right.
$$

Using the notations (similar to (8))

$$
\left\{\begin{array}{l}
\bar{D}_{1}^{(\mathrm{p})}=3 D_{\mathrm{m}}^{(\mathrm{p})} \\
\bar{D}_{2}^{(\mathrm{p})}=D_{33}^{(\mathrm{p})}
\end{array}\right.
$$

one may rewrite these relations in the form

$$
\left\{\begin{array}{l}
\bar{D}_{1}^{(\mathrm{p})}=\frac{\bar{D}_{1}}{c} \\
\bar{D}_{2}^{(\mathrm{p})}=\left(\frac{1}{c}-1\right) \bar{D}_{1}+\bar{D}_{2} .
\end{array}\right.
$$

As mentioned previously, the velocity field in the porous zone, $\mathbf{v}^{(\mathrm{p})}$, is chosen identical to Gurson (1977)'s "cylindrical velocity field":

$$
\left\{\begin{array}{l}
v_{r}^{(\mathrm{p})}(r)=\frac{3}{2} D_{\mathrm{m}}^{(\mathrm{p})} \frac{L^{2}}{r}-\frac{D_{33}^{(\mathrm{p})}}{2} r \\
v_{z}^{(\mathrm{p})}(z)=D_{33}^{(\mathrm{p})} z
\end{array}\right.
$$


while that in the sound zones, $\mathbf{v}^{(\mathrm{s})}$, is chosen so as to generate a uniform deviatoric strain rate:

$$
\left\{\begin{array}{l}
v_{r}^{(\mathrm{s})}(r)=D_{11} r \\
v_{z}^{(\mathrm{s})}(z)=-2 D_{11} z+K
\end{array}\right.
$$

In the second expression here $K$ is a constant adjusted so as to ensure continuity of the axial velocity component across the interface between the sound and porous parts, i.e. a no-jump condition $\llbracket v_{z} \rrbracket=0$. In terms of the kinematic parameters $\bar{D}_{1}, \bar{D}_{2}$, these velocity fields read:

$$
\begin{aligned}
& \left\{\begin{array}{l}
v_{r}^{(\mathrm{p})}(r)=\frac{1}{2}\left(\bar{D}_{1}^{(\mathrm{p})} \frac{L^{2}}{r}-\bar{D}_{2}^{(\mathrm{p})} r\right) \\
v_{z}^{(\mathrm{p})}(z)=\bar{D}_{2}^{(\mathrm{p})} z
\end{array}\right. \\
& \left\{\begin{array}{l}
v_{r}^{(\mathrm{s})}(r)=\frac{1}{2}\left(\bar{D}_{1}-\bar{D}_{2}\right) r \\
v_{z}^{(\mathrm{s})}(z)=\left(\bar{D}_{2}-\bar{D}_{1}\right) z+K
\end{array}\right.
\end{aligned}
$$

with $\bar{D}_{1}^{(\mathrm{p})}, \bar{D}_{2}^{(\mathrm{p})}$ given by (13). (Note that the overall field reduces to that used by Benzerga and Leblond (2014) to describe coalescence when $\left.D_{11}=0\right)$.

\section{Macroscopic criterion}

\subsection{Plastic dissipation}

Although strictly speaking, the macroscopic plastic dissipation corresponding to the velocity field defined by equations (17) and (18) is just an upper estimate of the true one, it will be denoted by the same symbol $\Pi(\mathbf{D})$ for convenience.

The macroscopic plastic dissipation $\Pi(\mathbf{D})$ consists of two volumetric contributions arising from the porous and sound zones, plus a surface contribution due to the interface:

$$
\Pi(\mathbf{D})=c \Pi^{(\mathrm{p})}+(1-c) \Pi^{(\mathrm{s})}+\Pi^{(\mathrm{i})}
$$


where

$$
\left\{\begin{array}{l}
\Pi^{(\mathrm{p})}=\frac{1}{\operatorname{vol}\left(\Omega^{(\mathrm{p})}\right)} \int_{\Omega^{(\mathrm{p})}} \sigma_{0} d_{\mathrm{eq}} d \Omega \\
\Pi^{(\mathrm{s})}=\frac{1}{\operatorname{vol}\left(\Omega^{(\mathrm{s})}\right)} \int_{\Omega^{(\mathrm{s})}} \sigma_{0} d_{\mathrm{eq}} d \Omega \\
\Pi^{(\mathrm{i})}=\frac{1}{\operatorname{vol}(\Omega)} \int_{\text {Interface }} \frac{\sigma_{0}}{\sqrt{3}}\left|\llbracket v_{\mathrm{t}} \rrbracket\right| d S,
\end{array}\right.
$$

$\llbracket v_{t} \rrbracket$ denoting the discontinuity of tangential velocity on the interface. These dissipations are calculated in Appendix C, and their final expressions are as follows:

- Volumetric dissipation in the porous zone:

$$
\Pi^{(\mathrm{p})}=\sigma_{0}\left|\bar{D}_{2}^{(\mathrm{p})}\right|\left[\frac{\sqrt{3}}{3} \frac{\bar{D}_{1}^{(\mathrm{p})}}{\bar{D}_{2}^{(\mathrm{p})}} \operatorname{Argsinh}\left(\frac{\sqrt{3}}{3} \frac{\bar{D}_{1}^{(\mathrm{p})}}{\bar{D}_{2}^{(\mathrm{p})}} u\right)-\sqrt{\frac{1}{u^{2}}+\frac{1}{3} \frac{\bar{D}_{1}^{(\mathrm{p}) 2}}{\bar{D}_{2}^{(\mathrm{p}) 2}}}\right]_{u=1}^{u=1 / \chi^{2}} .
$$

- Volumetric dissipation in the sound zone:

$$
\Pi^{(\mathrm{s})}=\sigma_{0}\left|\bar{D}_{1}-\bar{D}_{2}\right| \cdot
$$

- Surface dissipation at the interface:

$$
\Pi^{(\mathrm{i})}=\frac{\sigma_{0}}{3 \sqrt{3}} \frac{\chi^{3}-3 \chi+2}{W \chi}\left|\bar{D}_{1}\right| .
$$

\subsection{Regular, curved parts of the yield locus}

The regular parts of the yield locus are given by the parametric equations (10), which will now be made more explicit. It will be convenient to use stress components in the porous zone defined by

$$
\left\{\begin{array}{l}
\bar{\Sigma}_{1}^{(\mathrm{p})}=\Sigma_{11}^{(\mathrm{p})}=\frac{\partial \Pi^{(\mathrm{p})}}{\partial \bar{D}_{1}^{(\mathrm{p})}}\left(\bar{D}_{1}^{(\mathrm{p})}, \bar{D}_{2}^{(\mathrm{p})}\right) \\
\bar{\Sigma}_{2}^{(\mathrm{p})}=\Sigma_{33}^{(\mathrm{p})}-\Sigma_{11}^{(\mathrm{p})}=\frac{\partial \Pi^{(\mathrm{p})}}{\partial \bar{D}_{2}^{(\mathrm{p})}}\left(\bar{D}_{1}^{(\mathrm{p})}, \bar{D}_{2}^{(\mathrm{p})}\right) .
\end{array}\right.
$$

These expressions, which connect stress to strain rate components in the sole porous zone, are exactly identical to those connecting such components in a hollow, infinitely elongated cylinder, loaded in generalized plane strain. Therefore elimination of the ratio $\bar{D}_{1}^{(\mathrm{p})} / \bar{D}_{2}^{(\mathrm{p})}$ between equations $(24)_{1}$ and $(24)_{2}$ must lead to the same result as for an infinite hollow cylinder, that is to Gurson (1977)'s classical "cylindrical criterion" but for the sole porous part of the cell:

$$
\left(\frac{\Sigma_{33}^{(\mathrm{p})}-\Sigma_{11}^{(\mathrm{p})}}{\sigma_{0}}\right)^{2}+2 \chi^{2} \cosh \left(\sqrt{3} \frac{\Sigma_{11}^{(\mathrm{p})}}{\sigma_{0}}\right)-1-\chi^{4}=0
$$


where $\chi^{2}$ plays the role of the porosity.

By equation (19), the macroscopic stress components are given by:

$$
\left\{\begin{array}{l}
\bar{\Sigma}_{1}=\frac{\partial \Pi}{\partial \bar{D}_{1}}=c \frac{\partial \Pi^{(\mathrm{p})}}{\partial \bar{D}_{1}}+(1-c) \frac{\partial \Pi^{(\mathrm{s})}}{\partial \bar{D}_{1}}+\frac{\partial \Pi^{(\mathrm{i})}}{\partial \bar{D}_{1}} \\
\bar{\Sigma}_{2}=\frac{\partial \Pi}{\partial \bar{D}_{2}}=c \frac{\partial \Pi^{(\mathrm{p})}}{\partial \bar{D}_{2}}+(1-c) \frac{\partial \Pi^{(\mathrm{s})}}{\partial \bar{D}_{2}}+\frac{\partial \Pi^{(\mathrm{i})}}{\partial \bar{D}_{2}} .
\end{array}\right.
$$

Using equations (13) and (24), one transforms the expression of $\bar{\Sigma}_{1}$ into

$$
\begin{aligned}
\bar{\Sigma}_{1} & =c\left(\frac{\partial \Pi^{(\mathrm{p})}}{\partial \bar{D}_{1}^{(\mathrm{p})}} \frac{\partial \bar{D}_{1}^{(\mathrm{p})}}{\partial \bar{D}_{1}}+\frac{\partial \Pi^{(\mathrm{p})}}{\partial \bar{D}_{2}^{(\mathrm{p})}} \frac{\partial \bar{D}_{2}^{(\mathrm{p})}}{\partial \bar{D}_{1}}\right)+(1-c) \frac{\partial \Pi^{(\mathrm{s})}}{\partial \bar{D}_{1}}+\frac{\partial \Pi^{(\mathrm{i})}}{\partial \bar{D}_{1}} \\
& =\Sigma_{11}^{(\mathrm{p})}+(1-c)\left(\Sigma_{33}^{(\mathrm{p})}-\Sigma_{11}^{(\mathrm{p})}\right)+(1-c) \frac{\partial \Pi^{(\mathrm{s})}}{\partial \bar{D}_{1}}+\frac{\partial \Pi^{(\mathrm{i})}}{\partial \bar{D}_{1}} .
\end{aligned}
$$

Similarly,

$$
\begin{aligned}
\bar{\Sigma}_{2} & =c\left(\frac{\partial \Pi^{(\mathrm{p})}}{\partial \bar{D}_{1}^{(\mathrm{p})}} \frac{\partial \bar{D}_{1}^{(\mathrm{p})}}{\partial \bar{D}_{2}}+\frac{\partial \Pi^{(\mathrm{p})}}{\partial \bar{D}_{2}^{(\mathrm{p})}} \frac{\partial \bar{D}_{2}^{(\mathrm{p})}}{\partial \bar{D}_{2}}\right)+(1-c) \frac{\partial \Pi^{(\mathrm{s})}}{\partial \bar{D}_{2}}+\frac{\partial \Pi^{(\mathrm{i})}}{\partial \bar{D}_{2}} \\
& =c\left(\Sigma_{33}^{(\mathrm{p})}-\Sigma_{11}^{(\mathrm{p})}\right)+(1-c) \frac{\partial \Pi^{(\mathrm{s})}}{\partial \bar{D}_{2}}+\frac{\partial \Pi^{(\mathrm{i})}}{\partial \bar{D}_{2}} .
\end{aligned}
$$

By equations (22) and (23), the partial derivatives in equations (27) and (28) are given by:

$$
\left\{\begin{array}{l}
\frac{\partial \Pi^{(\mathrm{s})}}{\partial \bar{D}_{1}}=\sigma_{0} \operatorname{sgn}\left(\overline{\mathrm{D}}_{1}-\overline{\mathrm{D}}_{2}\right)=\sigma_{0} \operatorname{sgn}\left(\mathrm{D}_{11}\right) \\
\frac{\partial \Pi^{(\mathrm{s})}}{\partial \bar{D}_{2}}=\sigma_{0} \operatorname{sgn}\left(\overline{\mathrm{D}}_{2}-\overline{\mathrm{D}}_{1}\right)=-\sigma_{0} \operatorname{sgn}\left(\mathrm{D}_{11}\right) \\
\frac{\partial \Pi^{(\mathrm{i})}}{\partial \bar{D}_{1}}=\frac{\sigma_{0}}{3 \sqrt{3}} \frac{\chi^{3}-3 \chi+2}{W \chi} \operatorname{sgn}\left(\overline{\mathrm{D}}_{1}\right)=\frac{\sigma_{0}}{3 \sqrt{3}} \frac{\chi^{3}-3 \chi+2}{\mathrm{~W} \chi} \operatorname{sgn}\left(\mathrm{D}_{\mathrm{m}}\right) \\
\frac{\partial \Pi^{(\mathrm{i})}}{\partial \bar{D}_{2}}=0
\end{array}\right.
$$

where $\operatorname{sgn}(x)$ denotes the sign of $x$. Equations (27) and (28) then become

$$
\left\{\begin{array}{l}
\bar{\Sigma}_{1}=\Sigma_{11}=\Sigma_{11}^{(\mathrm{p})}+(1-c)\left(\Sigma_{33}^{(\mathrm{p})}-\Sigma_{11}^{(\mathrm{p})}\right)+A+B \\
\bar{\Sigma}_{2}=\Sigma_{33}-\Sigma_{11}=c\left(\Sigma_{33}^{(\mathrm{p})}-\Sigma_{11}^{(\mathrm{p})}\right)-A
\end{array}\right.
$$


where $\Sigma_{11}^{(\mathrm{p})}$ and $\Sigma_{33}^{(\mathrm{p})}-\Sigma_{11}^{(\mathrm{p})}$ are given by equations (24), and the quantities $A$ and $B$ by

$$
\left\{\begin{array}{l}
A=\sigma_{0}(1-c) \operatorname{sgn}\left(\mathrm{D}_{11}\right) \\
B=\frac{\sigma_{0}}{3 \sqrt{3}} \frac{\chi^{3}-3 \chi+2}{W \chi} \operatorname{sgn}\left(\mathrm{D}_{\mathrm{m}}\right) .
\end{array}\right.
$$

Equations (30) may be regarded as the parametric equations of the regular parts of the yield locus, in terms of the parameters $\bar{D}_{1}$ and $\bar{D}_{2}$ or $D_{11}$ and $D_{33}$. (Complementary details on this point are provided in Appendix D).

Alternatively, one may use equations (30) to express $\Sigma_{11}^{(\mathrm{p})}$ and $\Sigma_{33}^{(\mathrm{p})}-\Sigma_{11}^{(\mathrm{p})}$ in terms of $\Sigma_{11}$ and $\Sigma_{33}-\Sigma_{11}$, and insert the expressions found into the "yield criterion of the porous zone", equation (25). One thus gets the explicit equation of the regular parts of the overall yield locus:

$$
\Phi(\boldsymbol{\Sigma})=\left(\frac{\Sigma_{33}-\Sigma_{11}+A}{c \sigma_{0}}\right)^{2}+2 \chi^{2} \cosh \left(\sqrt{3} \frac{\Sigma_{11}-(1-c) \Sigma_{33}-A-c B}{c \sigma_{0}}\right)-1-\chi^{4}=0 .
$$

Note that four distinct zones of the yield locus, corresponding to different signs of the constants $A$ and $B$, are in fact described by this equation. Each of these zones is obviously curved.

\subsection{Singular, flat parts of the yield locus}

The presence of absolute values in the expressions (22) and (23) of $\Pi^{(\mathrm{s})}$ and $\Pi^{(\mathrm{i})}$ makes the total dissipation $\Pi$ a non-differentiable function of $\mathbf{D}$. Therefore there are some singular parts on the yield locus in addition to the regular ones. The study of these singular parts is the objective of this subsection.

Equations (10) being invalid on the singular parts of the yield locus, it is necessary to come back to the basic inequality (9). We begin by rewriting this inequality for both pairs $\left(\bar{D}_{1}, \bar{D}_{2}\right)$ and $\left(-\bar{D}_{1},-\bar{D}_{2}\right)$, so as to consider non-negative values of $\bar{D}_{2}$ only. The dissipation $\Pi$ being an even function of the pair $\left(\bar{D}_{1}, \bar{D}_{2}\right)$, one gets

$$
-\Pi\left(\bar{D}_{1}, \bar{D}_{2}\right) \leq \bar{\Sigma}_{1} \bar{D}_{1}+\bar{\Sigma}_{2} \bar{D}_{2} \leq \Pi\left(\bar{D}_{1}, \bar{D}_{2}\right) \quad, \quad \forall\left(\bar{D}_{1}, \bar{D}_{2}\right), \quad \bar{D}_{2} \geq 0
$$

We then divide inequalities (33) by $\bar{D}_{2}$, accounting for the property of positive homogeneity of degree 1 of the function $\Pi\left(\bar{D}_{1}, \bar{D}_{2}\right)$. Defining the dimensionless parameter

$$
\xi=\frac{\bar{D}_{1}}{\bar{D}_{2}}=\frac{3 D_{\mathrm{m}}}{D_{33}}
$$

one gets

$$
-g(\xi) \leq f(\xi) \leq g(\xi) \quad, \quad \forall \xi \quad,-\infty \leq \xi \leq+\infty
$$


where

$$
\left\{\begin{array}{l}
f(\xi)=\bar{\Sigma}_{1} \xi+\bar{\Sigma}_{2}=\Sigma_{11} \xi+\Sigma_{33}-\Sigma_{11} \\
g(\xi)=\Pi(\xi, 1)
\end{array}\right.
$$

Figure 2 shows, in a plane $(\xi, \zeta)$, a schematic diagram of the representative curves $\zeta=f(\xi)$, $\zeta=g(\xi), \zeta=-g(\xi)$ of the functions $f(\xi), g(\xi),-g(\xi)$. These curves possess the following properties:

- The curve $\zeta=f(\xi)$ is a straight line of slope $\bar{\Sigma}_{1}$ and ordinate at the origin $\bar{\Sigma}_{2}$.

- As a consequence of the convexity of the function $\Pi\left(\bar{D}_{1}, \bar{D}_{2}\right)$ established in Appendix E and the definition $(36)_{2}$ of the function $g(\xi)$, the curve $\zeta=g(\xi)$ is convex. Similarly, the curve $\zeta=-g(\xi)$ is concave.

- Since the expressions (22) and (23) of $\Pi^{(\mathrm{s})}$ and $\Pi^{(\mathrm{i})}$ are proportional to $\left|\bar{D}_{1}-\bar{D}_{2}\right|$ and $\left|\bar{D}_{1}\right|$ respectively, the expression of the function $g(\xi)$ contains terms proportional to $|\xi-1|$ and $|\xi|$; it follows that the curves $\zeta=g(\xi)$ and $\zeta=-g(\xi)$ have angular points at the positions $\xi=0$ and $\xi=1$. (At all other positions the curves are smooth).

- It is shown in Appendix $\mathrm{F}$ that the curves $\zeta=g(\xi)$ and $\zeta=-g(\xi)$ admit straight asymptotes at the limits $\xi= \pm \infty$; furthermore the asymptote to the curve $\zeta=g(\xi)$ at the point $\xi=+\infty$ (resp. $\xi=-\infty)$ coincides with the asymptote to the curve $\zeta=-g(\xi)$ at the point $\xi=-\infty$ (resp. $\xi=+\infty)$.

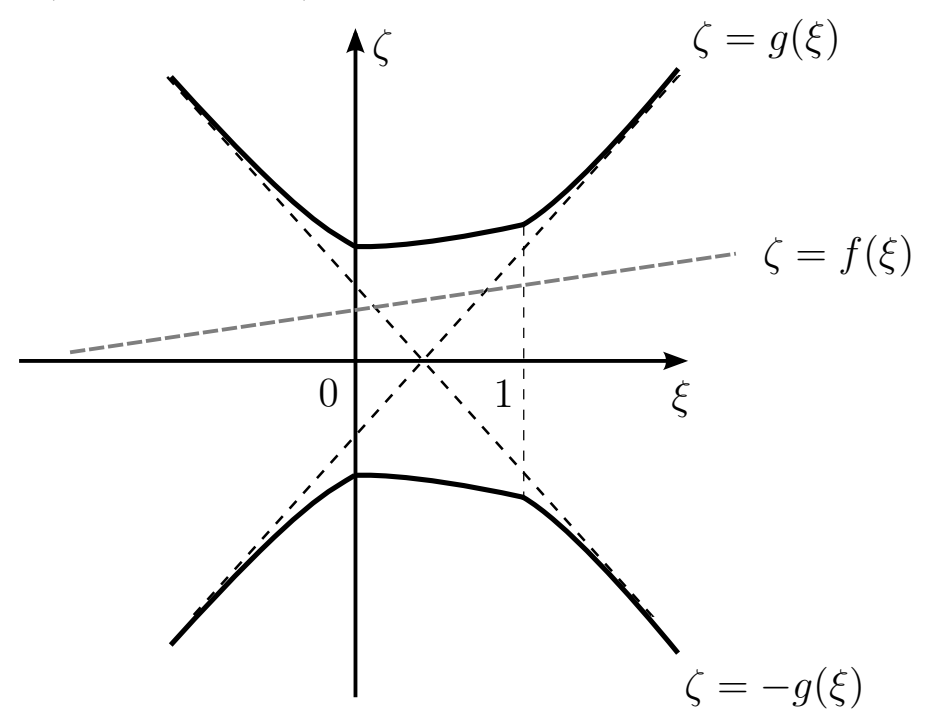

Fig. 2. Diagram of the representative curves of the functions $f(\xi), g(\xi),-g(\xi)$.

Inequalities (35) may then be discussed geometrically in a straightforward manner. These inequalities state that, in order for the pair $\left(\bar{\Sigma}_{1}, \bar{\Sigma}_{2}\right)$ to belong to the overall reversibility domain, the straight line $\zeta=f(\xi)$ must lie between the curves $\zeta=g(\xi)$ and $\zeta=-g(\xi)$ (see Fig. 2). Thus for this pair to lie on the overall yield locus (the boundary of the reversibility domain), the straight line $\zeta=f(\xi)$ must meet one of the curves $\zeta=g(\xi)$, $\zeta=-g(\xi)$ at some point, without crossing it. Since the yield locus is symmetric with respect to the origin, one may study only half of it. Thus one may consider the sole case where the straight line $\zeta=f(\xi)$ meets the curve $\zeta=g(\xi)$ without crossing it. ${ }^{1}$ It is clear from Fig. 2 that this may occur in three distinct cases:

1 In mathematical terms, we are then looking for the "subtangent lines" to the representative 
- The straight line $\zeta=f(\xi)$ is tangent to the curve $\zeta=g(\xi)$ at some point $P$ lying neither at $\xi=0$ nor at $\xi=1$ (Fig. 3a). In this situation the ordinate of the line at the origin $\bar{\Sigma}_{2}$ is a smooth function of its slope $\bar{\Sigma}_{1}$, which means that the point $\left(\bar{\Sigma}_{1}, \bar{\Sigma}_{2}\right)=\left(\Sigma_{11}, \Sigma_{33}-\Sigma_{11}\right)$ lies on one of the regular, curved parts of the yield locus studied in Subsection 4.2.

- The straight line $\zeta=f(\xi)$ meets the curve $\zeta=g(\xi)$ at $\xi=0$ and lies between the two tangents to this curve at this point (Fig. 3b). Then the ordinate of the line at the origin $\bar{\Sigma}_{2}=\Sigma_{33}-\Sigma_{11}$ is fixed, whereas its slope $\bar{\Sigma}_{1}=\Sigma_{11}$ may vary between specific bounds. In other words, the point $\left(\bar{\Sigma}_{1}, \bar{\Sigma}_{2}\right)=\left(\Sigma_{11}, \Sigma_{33}-\Sigma_{11}\right)$ lies on a flat portion (a segment) of the yield locus. The equation of this segment may be found by determining the coordinates of its endpoints, which may be done by taking the limits $\xi \rightarrow 0^{ \pm}$in the parametric equations (30), (D.1), (D.2), (D.3) of the regular parts of the yield locus; one thus gets

$$
\left\{\begin{array}{l}
\left|\Sigma_{11}+\sigma_{0}(1-c) \chi^{2}\right| \leq \frac{\sigma_{0}}{3 \sqrt{3}} \frac{\chi^{3}-3 \chi+2}{W \chi} \\
\Sigma_{33}-\Sigma_{11}=\sigma_{0}\left(1-c \chi^{2}\right) .
\end{array}\right.
$$

- The straight line $\zeta=f(\xi)$ meets the curve $\zeta=g(\xi)$ at $\xi=1$ and lies between the two tangents to this curve at this point (Fig. 3c). Then the ordinate of the line at $\xi=1$, that is $f(1)=\bar{\Sigma}_{1}+\bar{\Sigma}_{2}=\Sigma_{33}$ is fixed, whereas its slope $\bar{\Sigma}_{1}=\Sigma_{11}$ may vary between specific bounds. The point $\left(\bar{\Sigma}_{1}, \bar{\Sigma}_{2}\right)=\left(\Sigma_{11}, \Sigma_{33}-\Sigma_{11}\right)$ thus again lies on a flat portion of the yield locus, the equation of which may be found by determining its endpoints through consideration of the limits $\xi \rightarrow 1^{ \pm}$in equations (30), (D.1), (D.2), (D.3):

$$
\left\{\begin{array}{l}
\Sigma_{33}=\frac{\sigma_{0}}{\sqrt{3}}\left[\ln \left(\frac{1+\sqrt{1+3 \chi^{4}}}{3 \chi^{2}}\right)+2-\sqrt{1+3 \chi^{4}}\right]+\frac{\sigma_{0}}{3 \sqrt{3}} \frac{\chi^{3}-3 \chi+2}{W \chi} \\
\left|\Sigma_{33}-\Sigma_{11}-\frac{c \sigma_{0}}{\sqrt{3}}\left(2-\sqrt{1+3 \chi^{4}}\right)\right| \leq \sigma_{0}(1-c) .
\end{array}\right.
$$

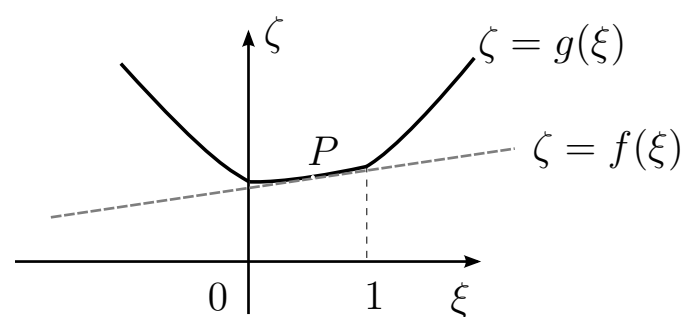

(a) General case: $\xi \neq 0, \xi \neq 1$

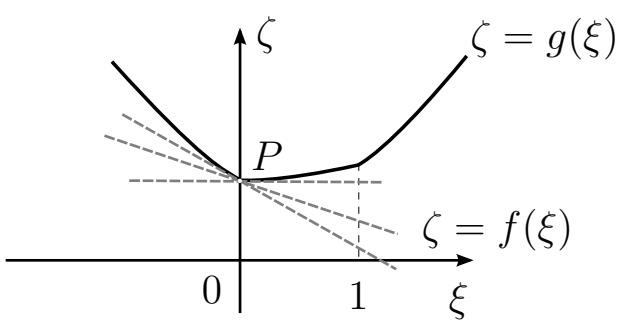

(b) Special case: $\xi=0$

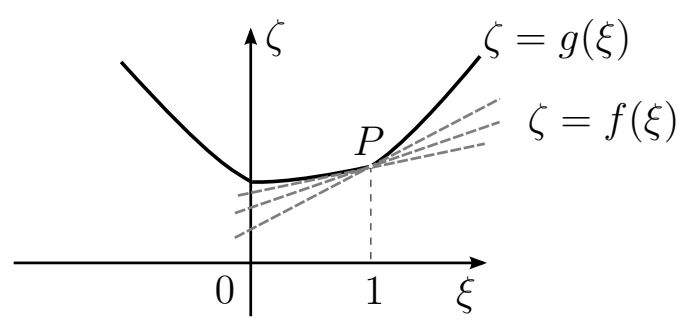

(c) Special case: $\xi=1$

Fig. 3. Qualitative study of the functions $f(\xi)$ corresponding to stresses lying on the yield locus.

$\overline{\text { curve } \zeta}=g(\xi)$ of the convex function $g(\xi)$. 
(It may be noted incidentally that if a gap existed between the tangent to the curve $\zeta=g(\xi)$ at $\xi=+\infty$ and the tangent to the curve $\zeta=-g(\xi)$ at $\xi=-\infty$, a third flat zone would exist on the yield locus; this flat zone would correspond to straight lines $\zeta=f(\xi)$ parallel to these tangents and lying between them. But such a gap does not exist since the tangents have been noticed above to coincide).

\subsection{Absence of corners on the yield locus}

The coexistence of curved and flat zones on the overall yield locus just evidenced suggests that corners might exist at the transition points between these zones. The object of this subsection is to show that no such corners exist. The key point in the proof is the fact that the complex expression $(21)$ of $\Pi^{(\mathrm{p})}$ implies that the function $g(\xi)$ is nonlinear on each of the intervals $(-\infty, 0),(0,1)$ and $(1,+\infty)$.

Consider for instance the transition between the singular part of the yield locus corresponding to straight lines $\zeta=f(\xi)$ meeting the curve $\zeta=g(\xi)$ at $\xi=0$, and the regular part corresponding to lines $\zeta=f(\xi)$ meeting the curve $\zeta=g(\xi)$ at some point $P$ having $\xi>0$. Since the singular part has $\bar{\Sigma}_{2}=\Sigma_{33}-\Sigma_{11}=$ const., showing that there is no corner on the yield locus is equivalent to showing that the derivative $\left(\mathrm{d} \bar{\Sigma}_{2} / \mathrm{d} \xi\right)\left(\xi=0^{+}\right)$is zero.

To establish such a property, consider the function $\tilde{g}(\xi)$ defined similarly to $g(\xi)$, but replacing $|\xi|$ with $\xi$ in its expression, that is replacing $\left|\bar{D}_{1}\right|$ with $\bar{D}_{1}$ in the expression (23) of $\Pi^{(\mathrm{i})}$ (see Fig. 4). This new function coincides with the old one $g(\xi)$ for $\xi \geq 0$ (not for $\xi<0$ !), but in contrast to it is perfectly smooth at $\xi=0$. Move the point $P$ of tangency of the straight line $\zeta=f(\xi)$ and the curve $\zeta=\tilde{g}(\xi)$ around the position $\xi=0$. Both the slope $\bar{\Sigma}_{1}$ and the ordinate at the origin $\bar{\Sigma}_{2}$ of the straight line vary (because the functions $g(\xi)$ and $\tilde{g}(\xi)$ are nonlinear), but it is clear from Fig. 4 that $\bar{\Sigma}_{2}$ reaches its maximum when $\xi=0$. Since it is a smooth function of $\xi$, the derivative $\mathrm{d} \bar{\Sigma}_{2} / \mathrm{d} \xi$ must be zero at $\xi=0^{+}$, which concludes the proof.

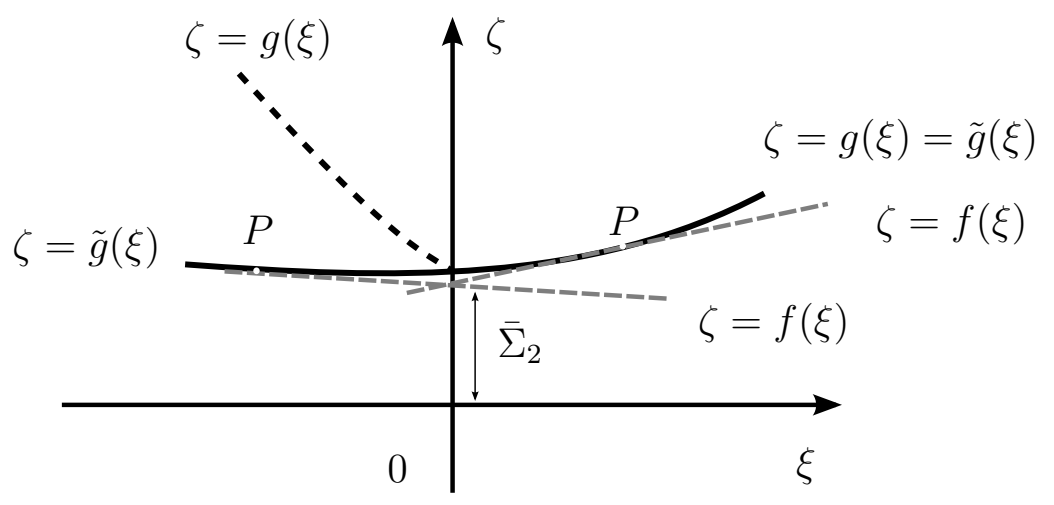

Fig. 4. Diagram of the representative curves of the functions $f(\xi)$ and $\tilde{g}(\xi)$.

It must however be stressed that this proof warrants the absence of corners on the sole approximate yield surface corresponding to the trial velocity fields envisaged. Nothing can be said at this stage about the true yield surface. 


\subsection{Summary}

The regular parts of the yield locus obey equation (32), with $A$ and $B$ given by equations (31). Note that each of these two quantities may take different signs, giving rise to four distinct portions of curved lines.

The singular parts of the yield locus also consist of four, now straight portions of lines (segments). Two of these segments are given by equations (37) and (38). The other two are obtained by accounting for the symmetry of the yield locus with respect to the origin, that is by replacing $\Sigma_{11}$ and $\Sigma_{33}$ with $-\Sigma_{11}$ and $-\Sigma_{33}$ in these equations.

\section{Assessment of the theoretical model}

\subsection{Principle of the simulations}

The objective of this section is to provide a numerical assessment of the theoretical criterion derived above. To this end, the limit-analysis problem is solved using the customary finite element method including elasticity. This is done by imposing a large load step with no geometry update; indeed it has been shown by Madou and Leblond (2012a) that under such conditions, the time-discretized equations of the finite element problem are equivalent to those of limit-analysis, provided that the local "projection problem" (plastic correction of the elastic stress predictor) is solved using an implicit (backward Euler) algorithm.

The numerical simulations are performed using the finite element code SYSTUS ${ }^{\circledR}$ developed by ESI Group. 2D axisymmetric meshes are used since a cylindrical cell was considered in the derivation of the theoretical model. The boundary conditions are represented schematically in Fig. 5. Axisymmetric loadings are considered $\left(\Sigma_{11}=\Sigma_{22} \neq 0\right.$, $\Sigma_{33} \neq 0$ and $\Sigma_{i j}=0$ otherwise); the macroscopic stresses $\Sigma_{11}$ and $\Sigma_{33}$ are obtained from their microscopic counterparts through the formula:

$$
\Sigma_{i j}=\frac{1}{\operatorname{vol}(\Omega)} \int_{\Omega-\omega} \sigma_{i j} d \Omega
$$

It must be emphasized that these numerical simulations are performed for exactly the same cells, with a single embedded void, and exactly the same boundary conditions, as the analytical calculations. We do not consider large cells containing many voids distributed more or less randomly, because such cells, although well fit to the study of void growth, are not adapted to that of coalescence; see more details on this topic in Appendix B. 


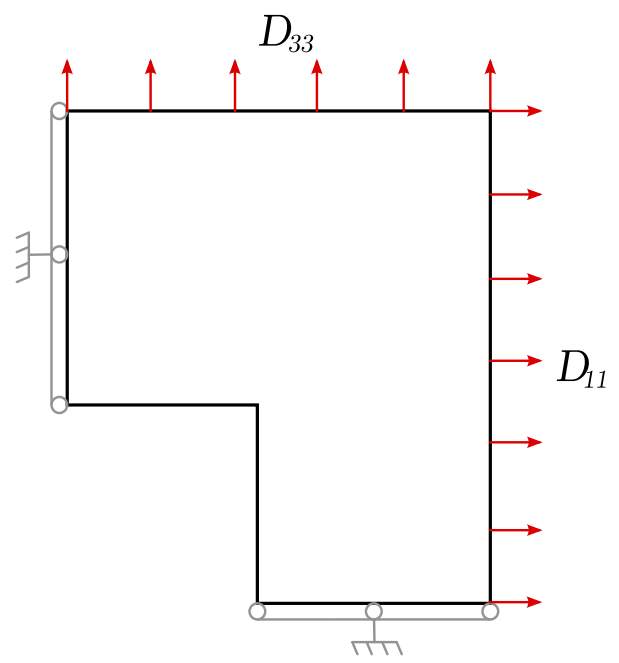

Fig. 5. Boundary conditions considered for the study of the yield locus of a cylindrical hollow cell.

\subsection{Numerical results}

Results for the yield locus of a cylindrical hollow cell are provided in Fig. 6 for a cell aspect ratio $\lambda=1$, a void aspect ratio $W=1$ and two values of the ligament parameter, $\chi=0.4$ and 0.6 . The yield surfaces are represented in the plane $\left(\Sigma_{\mathrm{m}} / \sigma_{0},\left(\Sigma_{33}-\Sigma_{11}\right) / \sigma_{0}\right)$, where $\Sigma_{\mathrm{m}}=\frac{1}{3}\left(2 \Sigma_{11}+\Sigma_{33}\right)$ is the mean normal stress. Three types of results are plotted:

- The numerical yield locus obtained through finite element limit-analysis.

- A "hybrid" criterion obtained by truncating Gurson (1977)'s yield locus for a hollow sphere ${ }^{2}$ with the same porosity as the cylindrical cell considered, by the straight line of equation $(38)_{1}$, corresponding to the coalescence criterion of Benzerga and Leblond (2014). The "truncation" means that for any loading direction, the estimate of the limit-load provided by Gurson (1977)'s criterion is compared to that given by equation $(38)_{1}$, and the lower estimate is selected, in the spirit of limit-analysis.

- The theoretical "unified" criterion obtained in Section 4.

Prior to discussing the results shown in Fig. 6, it is necessary to mention that the model results have been obtained by introducing, in both models, a heuristic parameter $\mu$ slightly modifying the value of the ligament parameter $\chi=R / L$ governing the value of $\Sigma_{33}$ at coalescence: $\chi$ has been replaced by $\chi^{*}=\mu \chi$. The chosen values of $\mu$ are in fact quite close to unity: 1.12 for $\chi=0.4$ and 1.065 for $\chi=0.6{ }^{3}$ The explanation of this adjustment is as follows. It has been noted, and discussed at length by Morin et al. (2015a), that the axial stress $\Sigma_{33}$ for the coalescence stage predicted by Benzerga and Leblond (2014)'s original criterion slightly overestimates that obtained numerically. But the discrepancy is of little interest here, our goal being essentially to compare the two analytical criteria.

$\overline{2}$ Gurson (1977)'s "cylindrical" criterion cannot be used because the cylindrical void implied extends up and down to the top and bottom surfaces of the cell, in contrast to the truncated one considered here.

3 Note however that the introduction of $\mu$ slightly destroys the upper bound character of the theoretical criterion, as can be seen in Fig. 6 for $\chi=0.6$. 


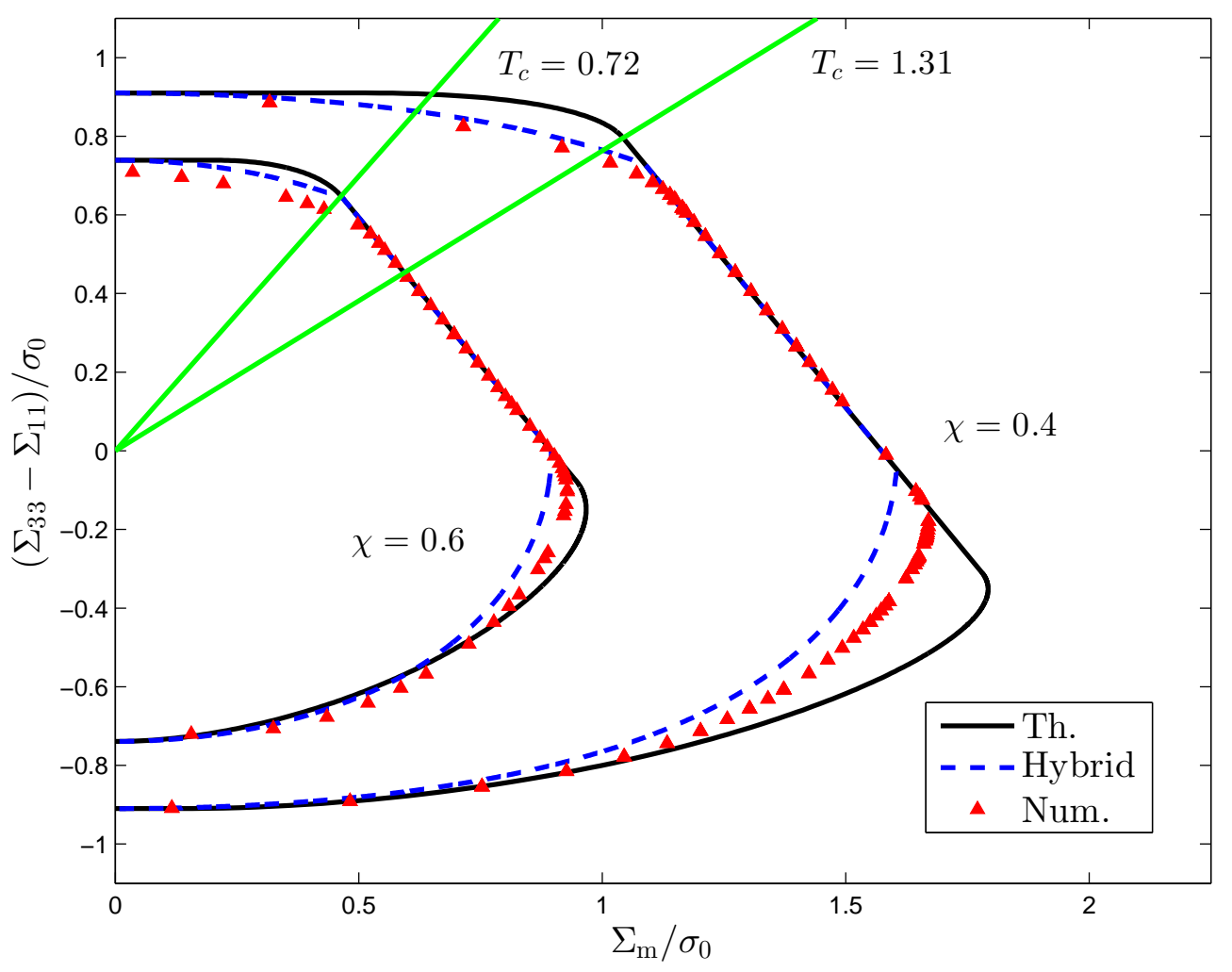

Fig. 6. Yield surfaces for $\lambda=1, W=1$ and two values of $\chi$.

The introduction of the parameter $\mu$ permits to remove it. The modification is quite analogous, in the context of void coalescence, to Tvergaard (1981)'s heuristic proposal of multiplication of the porosity by some adjustable parameter $q$, in the context of void growth.

\subsection{Discussion}

\subsubsection{Preliminary remark on the influence of void shape}

By the expression "influence of void shape" is meant here the effect of the type (cylindrical/spheroidal) of void considered for a given value of the void aspect ratio (ratio of the vertical to horizontal dimensions of the void); not the effect of the void aspect ratio for a given type of void. It has been shown by Morin et al. (2015a), through numerical finite-analyses of elementary cells, that the influence of void shape upon the computed limit loads, with this definition, is minor. This supports the idea that the assumption of cylindrical voids of finite height, in both theoretical analyses and numerical computations of void coalescence, is only mildly restrictive. 


\subsubsection{Flat and curved parts of the yield locus}

The three criteria represented in Fig. 6 exhibit the same basic features: they are all composed of curved and flat portions.

- The curved parts correspond to the growth stage, that is to solutions for which the plastic strain rate is diffuse in the entire cell.

- By way of contrast, the flat part on the right, of equation $\Sigma_{33}=$ const. where the constant depends on $\chi$ and $W$, corresponds to the coalescence stage, that is to solutions for which the plastic strain rate is concentrated within the inter-void ligament. To understand why coalescence gives rise to such a straight segment on the yield locus, it suffices to note that the presence of rigid zones above and below the void during this phase implies that the horizontal components of the macroscopic strain rate become nil. This in turn implies, via the normality property of the macroscopic flow rule, that the overall yield function no longer depends on the horizontal stress $\Sigma_{11}$.

Straight lines of constant triaxiality $T=\Sigma_{m} /\left|\Sigma_{33}-\Sigma_{11}\right|$, also represented in Fig. 6, permit to appreciate the value of the "critical" triaxiality $T_{c}$ above which coalescence sets in. This triaxiality may be observed to depend on the geometrical parameters of the cell: for $\lambda=1$ and $W=1$ (as considered in the figure), $T_{c}=1.31$ for $\chi=0.4$ and $T_{c}=0.72$ for $\chi=0.6$. This last example illustrates the fact that coalescence may occur for relatively low values of the triaxiality; also, the good agreement which may be observed between numerical results and model predictions even in this case shows that the models are not limited to conditions of high triaxiality (although they are limited to axisymmetric loadings).

\subsubsection{Comparison of theoretical criteria}

On the "coalescence line", the predictions of the hybrid and unified models coincide. This is not surprising since they are based on the same trial velocity field. Some differences, however, appear in the regions of the yield locus corresponding to the growth stage and in the "transition regions". These differences highlight the specific features of each approach.

Hybrid criterion. The advantages of this criterion are as follows:

- For the growth stage, the yield locus predicted by the hybrid criterion is closer to the numerical one than that predicted by the unified criterion, at least for major axial stresses $\left(\Sigma_{33}>\Sigma_{11}=\Sigma_{22}\right)$.

- The hybrid criterion may benefit, in more advanced versions, from the sophisticated extensions of Gurson's criterion for the growth stage, including e.g. void shape effects and plastic anisotropy; see for instance (Gologanu et al., 1993, 1994, 1997; Benzerga and Besson, 2001; Benzerga et al., 2004; Monchiet et al., 2008; Keralavarma and Benzerga, 2010; Madou and Leblond, 2012a,b, 2013; Madou et al., 2013; Morin et al., 2015b). Also, it may be applied to general loading conditions.

However the hybrid criterion also has disadvantages:

- Since the reversibility domain is the intersection of two reversibility domains derived 
from distinct, independent sources, there are some inevitable corners on the yield locus. The numerical simulations, however, do not exhibit such corners (see Fig. 6). Their presence in the hybrid yield locus may generate spurious instability and bifurcation phenomena in finite element computations of specimens or structures using this type of model.

- The theoretical foundations of the hybrid criterion are less appealing than those of the unified one, since the intersecting reversibility domains do not pertain to the same geometry (a hollow sphere was considered in Gurson (1977)'s work for the growth phase, versus a hollow cylinder in Benzerga and Leblond (2014)'s work and here for the coalescence phase).

Unified criterion. The advantages of the unified criterion are as follows:

- The yield locus has no corners, in agreement with the numerical results, even though regions of high curvature may be observed in Fig. 6 at the transition points between the regions corresponding to the growth and coalescence stages.

- Growth and coalescence are described with a single class of velocity fields, thus emphasizing the basic unity of the two stages and the possibility of their unified description.

However, the unified model also suffers from some disadvantages, at least in the version presented here:

- The presence on the yield locus of a flat part of equation $\Sigma_{33}-\Sigma_{11}=$ const. for low triaxialities, arising from the velocity discontinuity across the interface between the sound and porous parts of the cell, implies that the predicted porosity rate is nil under such conditions, which contradicts both intuition and numerical results. This observation emphasizes the shortcomings of discontinuous trial velocity fields, and raises the issue of the implications of the potential use of more realistic, continuous velocity fields upon the predicted yield locus. This issue was in part tackled by Morin et al. (2015a) but only for the coalescence phase.

- The criterion is limited, even for the growth phase, to axisymmetric loadings and cylindrical voids.

\subsubsection{Remarks on flatness, anisotropy and periodicity}

The flatness of some parts of the overall yield locus has been noted to arise, via the macroscopic normality property, from the condition of zero lateral straining, $D_{11}=D_{22}=$ 0 , during the coalescence phase. This condition ascribes a special role to the axial direction $x_{3}$ and hence also implies an anisotropy of the yield locus. It is intimately tied to (i) the assumed periodicity of the distribution of voids; (ii) the orientation of the lattice of voids; and (iii) the orientation of the (assumedly axisymmetric) loading with respect to this lattice. The predicted anisotropy of the yield locus raises the issue of the relevance of the periodicity hypothesis, since "real" materials are expected to be at least initially (before the onset of coalescence) isotropic - the distribution of voids being more or less random.

A short discussion of this problem is provided at the end of Appendix B, including heuristic remedies aimed at "adapting" the theoretical yield locus for a periodic distribution of voids 
to more realistic distributions. It is for instance suggested to vary, at every given point, the orientation of the hypothetic periodic lattice of voids and, in the spirit of limit-analysis, select that orientation leading to the lowest estimate of the limit-load. Such an approach will of course erase the flat parts of the yield locus, since it implies considering now the reversibility domain as the intersection of infinitely many domains having each flat parts, but with various orientations. It will also result in an again isotropic (though perhaps hard to define analytically) yield locus.

It must however be noted that the yield locus will become anisotropic as soon as coalescence sets in. Indeed onset of coalescence implies selection of a specific orientation of the planar band of localization, which destroys isotropy onwards.

\section{Conclusion}

A framework has been laid out to develop macroscopic yield criteria for porous ductile solids accounting for both void growth and coalescence. While the framework is general, closed form solutions have been obtained in the specific case of cylindrical cells containing cylindrical voids of a given aspect ratio and subjected to axisymmetric loading conditions. The main conclusions are as follows:

- The "unified" model proposed provides a unique description of both growth and coalescence of voids, and a rigorous upper bound to the exact locus. The growth phase corresponds to portions of curved lines on this locus and the coalescence phase to portions of flat ones.

- There are no corners on the yield surface predicted by the unified model, in agreement with the results of numerical computations of the cylindrical cells considered. This surface however exhibits regions of high curvature at the transition points between the sectors corresponding to growth and coalescence. The implications of such a feature on plastic flow localization at the macroscopic scale remain to be investigated.

- The proposed model is a first step toward a unified description of void growth and coalescence. Although it is not limited to high triaxialities ${ }^{4}$, its main limitation is the restriction to axisymmetric loadings only. An extended model incorporating shear loading states is currently under development.

\section{Acknowledgments}

AAB acknowledges the support of the National Science Foundation under Grant Number CMMI-1405226.

\footnotetext{
4 This also applies to the "hybrid" model, which can be improved for low triaxialities by using a suitable extension of Gurson's model accounting for void shape effects to describe void growth prior to coalescence; see the work of Gologanu et al. (1997) for instance.
} 


\section{A Coalescence and strain localization theory}

The connection between coalescence and strain localization seems obvious, at least for coalescence through internal necking, since this necking occurs through concentration of the strain rate within the ligaments separating neighboring voids. However, deciding whether or not coalescence can simply be described using the standard theory of strain localization is a less trivial question than may seem at first sight.

First, it must be clear that coalescence does not correspond to strain localization at the microscopic scale. In Koplik and Needleman (1988)'s numerical simulations, concentration of the strain rate within the horizontal intervoid ligaments occurred although the microscopic constitutive behavior was governed by a standard von Mises model with positive (isotropic) strain hardening, for which strain localization is known to be impossible. Coalescence corresponds to concentration of the strain rate within a layer of thickness comparable to the void size, not to infinite concentration.

It may then seem natural to interpret coalescence as a localization mechanism at the larger scale of some homogenized model incorporating the presence of voids. Perrin (1992) tried to apply this idea to Koplik and Needleman (1988)'s numerical simulations of coalescence, by schematizing their "representative cells" as elementary volumes obeying Gurson (1977)'s model and using Rudnicki and Rice (1975)'s results on localization in pressure-sensitive materials, as specialized to the case of Gurson's model by Yamamoto (1978). ${ }^{5}$ He observed that Gurson (1977)'s model did predict strain localization within planar layers parallel to the intervoid ligaments, in agreement with Koplik and Needleman (1988)'s observations, but for values of the porosity far greater than those reported by these authors at the onset of coalescence.

The natural interpretation of this failure is that Gurson (1977)'s model is basically inadequate to predict coalescence, because the trial velocity fields it is derived from correspond to widely spread strain rate fields, in contrast to the concentrated fields corresponding to coalescence. In other words, because of the "smooth" velocity fields it considers, this model correctly depicts void growth, but is not fit to depict the concentration of the strain rate at a scale smaller than the void spacing.

What precedes does not mean that trying to predict coalescence by applying the theory of strain localization to some homogenized model is a hopeless task. It just emphasizes the need, in order to do so, of a model incorporating the mesoscopic mechanism of coalescence, that is concentration of the strain rate at a scale of the order of the void size, not the void spacing. This means, if the model is derived from limit-analysis, that this analysis must incorporate trial velocity fields depicting such a concentration. Since Gurson (1977)'s model does not satisfy this condition, it is necessary to complete it by considering velocity fields of this type, which is precisely the topic of this paper.

\footnotetext{
5 The version of the model used was of course the original one, not incorporating Tvergaard and Needleman (1984)'s heuristic modelling of coalescence through some artificial "effective porosity".
} 


\section{B Coalescence and homogenization theory}

Standard homogenization theories are based on a fundamental assumption of separation of scales: the typical size of heterogeneities is supposed to be much smaller than the distance over which the macroscopic mechanical fields vary significantly. In the case of voids in porous plastic materials, this means that the macroscopic stress and strain rate fields must vary only over distances encompassing many voids. This is generally true during the void growth phase. However, when coalescence of voids sets in, the porosity generally concentrates in a small zone containing only a few voids, within which the macroscopic stresses and strain rates vary significantly; the typical situation is that of a coalescence region involving only two or three voids ahead of a crack tip. Homogenization theories do not, strictly speaking, apply to such situations.

The small size of the coalescence region leaves no choice, in theoretical studies of the phenomenon, but to consider "elementary cells" containing only a few voids. Such studies may be classified as "analyses of elementary mechanisms" but they do not, strictly speaking, pertain to homogenization theories. The basic relations of homogenization connecting local and overall quantities are nevertheless retained: the macroscopic stresses and strain rates are still identified to the average values of the corresponding microscopic quantities over the cell considered, and similarly for the macroscopic and microscopic power densities.

Various conditions may be envisaged a priori on the boundary of the elementary cell; unfortunately, for the small cells to be considered, these boundary conditions give rise to different overall responses. This immediately raises the problem of choosing the "right" ones. Classical Hill-Mandel (Hill, 1967; Mandel, 1964) conditions of homogeneous boundary strain rate cannot be used to study coalescence because they cannot accommodate rigid regions extending to the boundary, thereby precluding the possibility of concentration of the strain rate within localized zones. Hill-Mandel conditions of homogeneous boundary stresses generally lead to overly compliant overall responses in problems of porous plastic solids. Therefore the only choice left - adopted in all analyses of coalescence up to now and again in the present work - is that of periodic boundary conditions.

It must however be stressed that this choice puts considerable, and unfortunately physically unrealistic restrictions on the distributions of voids considered. This means that the approximate overall yield criteria and flow rules obtained with the periodicity hypothesis must necessarily be "adapted" in some way before they can be applied to practical situations often involving clustered distributions of voids.

There are several ways to achieve such an adaptation. Two typical solutions are as follows:

- Modify the model, developed for a fixed orientation of the assumed periodic lattice of voids, by considering arbitrary possible orientations of this lattice, and selecting the "optimal" one. This has been done by Leblond and Mottet (2008) in the spirit of limitanalysis, using a specific model to estimate the limit-load corresponding to a given orientation and minimizing this load.

- In a more numerical, "finite-element-oriented" approach, mesh the region ahead of the 
crack tip in a regular way, e.g. with cubical elements with size of the order of the mean void spacing; use the model derived with the periodicity hypothesis in each of these elements; but vary the orientation of the periodic lattice from one element to another.

Note that the first solution amounts to performing an "ensemble" average over different possible "realizations" of the material, whereas the second amounts to performing a spatial average. Of course, both of them still make reference to a non-existent periodic lattice of voids, although they consider random variations of the orientation of this lattice within the model itself or within the finite element mesh. Therefore they do not represent a rigorous solution to the problem of coalescence of voids in "real" porous plastic solids often involving clustered distributions of voids. But approaches of this kind represent "the best that one can do" at present, in the absence of a "clean" homogenization theory of coalescence.

\section{Volumetric and surfacic plastic dissipations}

\section{C.1 Porous zone}

In the porous part, the expression of the equivalent strain rate associated to the velocity field defined by equations (17) reads

$$
d_{\mathrm{eq}}^{(\mathrm{p})}=\sqrt{\bar{D}_{2}^{(\mathrm{p}) 2}+\frac{1}{3} \bar{D}_{1}^{(\mathrm{p}) 2} \frac{L^{4}}{r^{4}}} .
$$

Therefore the plastic dissipation in the porous part is given by

$$
\begin{aligned}
\Pi^{(\mathrm{p})} & =\frac{1}{\operatorname{vol}\left(\Omega^{(\mathrm{p})}\right)} \int_{\Omega^{(\mathrm{p})}} \sigma_{0} d_{\mathrm{eq}}^{(\mathrm{p})} d \Omega \\
& =\frac{\sigma_{0}}{2 \pi h L^{2}} 4 \pi h\left|\bar{D}_{2}^{(\mathrm{p})}\right| \int_{R}^{L} \sqrt{1+\frac{1}{3} \frac{\bar{D}_{1}^{(\mathrm{p}) 2}}{\bar{D}_{2}^{(\mathrm{p}) 2}} \frac{L^{4}}{r^{4}}} r \mathrm{~d} r .
\end{aligned}
$$

Using the change of variable $u=L^{2} / r^{2}$, one finds the final expression of this dissipation:

$$
\begin{aligned}
\Pi^{(\mathrm{p})} & =\sigma_{0}\left|\bar{D}_{2}^{(\mathrm{p})}\right| \int_{1}^{1 / \chi^{2}} \sqrt{1+\frac{1}{3} \frac{\bar{D}_{1}^{(\mathrm{p}) 2}}{\bar{D}_{2}^{(\mathrm{p}) 2}} u^{2}} \frac{\mathrm{d} u}{u^{2}} \\
& =\sigma_{0}\left|\bar{D}_{2}^{(\mathrm{p})}\right|\left[\frac{\sqrt{3}}{3} \frac{\bar{D}_{1}^{(\mathrm{p})}}{\bar{D}_{2}^{(\mathrm{p})}} \operatorname{Argsinh}\left(\frac{\sqrt{3}}{3} \frac{\bar{D}_{1}^{(\mathrm{p})}}{\bar{D}_{2}^{(\mathrm{p})}} u\right)-\sqrt{\left.\frac{1}{u^{2}}+\frac{1}{3} \frac{\bar{D}_{1}^{(\mathrm{p}) 2}}{\bar{D}_{2}^{(\mathrm{p}) 2}}\right]_{u=1}^{u=1 / \chi^{2}} .} .\right.
\end{aligned}
$$




\section{C.2 Sound zone}

In the sound part, the expression of the equivalent strain rate reads

$$
d_{\mathrm{eq}}^{(\mathrm{s})}=\left|D_{33}^{(\mathrm{s})}\right|=\left|\bar{D}_{1}-\bar{D}_{2}\right|
$$

so that the macroscopic plastic dissipation is simply given by

$$
\Pi^{(\mathrm{s})}=\sigma_{0}\left|\bar{D}_{1}-\bar{D}_{2}\right| \text {. }
$$

\section{C.3 Interface}

The surface dissipation on the interface is given by

$$
\Pi^{(\mathrm{i})}=\frac{1}{\operatorname{vol}(\Omega)} \int_{\text {Interface }} \frac{\sigma_{0}}{\sqrt{3}}\left|\llbracket v_{\mathrm{t}} \rrbracket\right| d S
$$

where

$$
\llbracket v_{\mathrm{t}} \rrbracket=v_{r}^{(\mathrm{s})}(r)-v_{r}^{(\mathrm{p})}(r)=\frac{\bar{D}_{1}}{2 c}\left(r-\frac{L^{2}}{r}\right)
$$

The expression of $\Pi^{(i)}$ consequently reads, accounting for the presence of two surfaces of discontinuity:

$$
\begin{aligned}
\Pi^{(\mathrm{i})} & =\frac{\sigma_{0}}{\sqrt{3}} \frac{2}{2 \pi H L^{2}} \frac{\left|\bar{D}_{1}\right|}{2 c} \int_{R}^{L}\left(L^{2}-r^{2}\right) 2 \pi \mathrm{d} r \\
& =\frac{\sigma_{0}}{\sqrt{3} h L^{2}}\left|\bar{D}_{1}\right|\left(\frac{2 L^{3}}{3}-L^{2} R+\frac{R^{3}}{3}\right) \\
& =\frac{\sigma_{0}}{3 \sqrt{3}} \frac{\chi^{3}-3 \chi+2}{W \chi}\left|\bar{D}_{1}\right| .
\end{aligned}
$$

\section{Parametric equations of the regular parts of the yield locus}

Further details are provided here on the parametric representation (30) of the regular parts of the yield locus.

Since the overall yield locus is symmetric with respect to the origin, it suffices to define only half of it, and thus assume $\bar{D}_{2}=D_{33} \geq 0$. One may also define the dimensionless parameter

$$
\xi^{(\mathrm{p})}=\frac{\bar{D}_{1}^{(\mathrm{p})}}{\bar{D}_{2}^{(\mathrm{p})}}=\frac{3 D_{\mathrm{m}}^{(\mathrm{p})}}{D_{33}^{(\mathrm{p})}}=\frac{\xi}{(1-c) \xi+c}
$$


where equations (13), (14) and (34) have been used. Then, by equations (21) and (24), the quantities $\Sigma_{11}^{(\mathrm{p})}$ and $\Sigma_{33}^{(\mathrm{p})}-\Sigma_{11}^{(\mathrm{p})}$ in the expressions (30) of $\Sigma_{11}$ and $\Sigma_{33}-\Sigma_{11}$ are given as functions of the parameter $\xi$ by

$$
\left\{\begin{array}{l}
\Sigma_{11}^{(\mathrm{p})}=\frac{\sigma_{0}}{\sqrt{3}} \operatorname{sgn}((1-c) \xi+c)\left[\operatorname{Argsinh}\left(\frac{\xi^{(\mathrm{p})}}{\sqrt{3} \chi^{2}}\right)-\operatorname{Argsinh}\left(\frac{\xi^{(\mathrm{p})}}{\sqrt{3}}\right)\right] \\
\Sigma_{33}^{(\mathrm{p})}-\Sigma_{11}^{(\mathrm{p})}=\sigma_{0} \operatorname{sgn}((1-c) \xi+c)\left[\sqrt{\frac{\xi^{(\mathrm{p}) 2}}{3}+1}-\sqrt{\frac{\xi^{(\mathrm{p}) 2}}{3}+\chi^{4}}\right]
\end{array}\right.
$$

with $\xi^{(\mathrm{p})}$ given by (D.1). Also, by equations (31) and (34), the constants $A$ and $B$ are given in terms of $\xi$ by

$$
\left\{\begin{array}{l}
A=\sigma_{0}(1-c) \operatorname{sgn}(\xi-1) \\
B=\frac{\sigma_{0}}{3 \sqrt{3}} \frac{\chi^{3}-3 \chi+2}{W \chi} \operatorname{sgn}(\xi)
\end{array}\right.
$$

\section{E Convexity of the macroscopic plastic dissipation}

In this Appendix, it becomes exceptionally necessary to distinguish between the true plastic dissipation $\Pi$, defined by equation (5) with $\mathbf{v}$ spanning the whole set $\mathcal{K}(\mathbf{D})$ of incompressible velocity fields kinematically admissible with $\mathbf{D}$, and its upper estimate $\Pi^{+}$, defined by the same equation but with $\mathbf{v}$ spanning some subset $\mathcal{K}^{\prime}(\mathbf{D})$ of $\mathcal{K}(\mathbf{D})$.

The function to be proved convex is $\Pi^{+}\left(\bar{D}_{1}, \bar{D}_{2}\right)$ (noted simply $\Pi\left(\bar{D}_{1}, \bar{D}_{2}\right)$ in the main text). The proof will proceed in three steps.

Step 1. We first note that the microscopic plastic dissipation $\pi(\mathbf{d})=\sigma_{0} d_{\mathrm{eq}}$ is a convex function of the (traceless) tensor $\mathbf{d}$, since it is proportional to its Euclidian norm. ${ }^{6}$

Step 2. Consider now two incompressible velocity fields $\mathbf{v}^{1} \in \mathcal{K}\left(\mathbf{D}^{1}\right), \mathbf{v}^{2} \in \mathcal{K}\left(\mathbf{D}^{2}\right)$ and some real number $\theta \in[0,1]$. Then the field $(1-\theta) \mathbf{v}^{1}+\theta \mathbf{v}^{2}$ is incompressible, and

$$
\int_{\Omega} \pi\left[(1-\theta) \mathbf{d}^{1}+\theta \mathbf{d}^{2}\right] d \Omega \leq(1-\theta) \int_{\Omega} \pi\left(\mathbf{d}^{1}\right) d \Omega+\theta \int_{\Omega} \pi\left(\mathbf{d}^{2}\right) d \Omega
$$

by Step 1 . Now since the field $(1-\theta) \mathbf{v}^{1}+\theta \mathbf{v}^{2}$ is kinematically admissible with $(1-\theta) \mathbf{D}^{1}+$ $\theta \mathbf{D}^{2}$, it follows from the definition (5) of $\Pi(\mathbf{D})$ that

$$
\Pi\left[(1-\theta) \mathbf{D}^{1}+\theta \mathbf{D}^{2}\right] \leq \int_{\Omega} \pi\left[(1-\theta) \mathbf{d}^{1}+\theta \mathbf{d}^{2}\right] d \Omega,
$$

$\overline{6}$ This property in fact holds for any yield criterion, not only that of von Mises, as a consequence of the definition of $\pi(\mathbf{d})$ as the Legendre transform of the indicator function of the convex set of reversibility. 
so that, by the preceding inequality,

$$
\Pi\left[(1-\theta) \mathbf{D}^{1}+\theta \mathbf{D}^{2}\right] \leq(1-\theta) \int_{\Omega} \pi\left(\mathbf{d}^{1}\right) d \Omega+\theta \int_{\Omega} \pi\left(\mathbf{d}^{2}\right) d \Omega .
$$

Since this is true for all incompressible velocity fields $\mathbf{v}^{1} \in \mathcal{K}\left(\mathbf{D}^{1}\right), \mathbf{v}^{2} \in \mathcal{K}\left(\mathbf{D}^{2}\right)$, this implies, taking the infimum of the right-hand side, that

$$
\Pi\left[(1-\theta) \mathbf{D}^{1}+\theta \mathbf{D}^{2}\right] \leq(1-\theta) \Pi\left(\mathbf{D}^{1}\right)+\theta \Pi\left(\mathbf{D}^{2}\right),
$$

which shows that the function $\Pi(\mathbf{D})$ is convex.

Step 3. The proof of the convexity of the function $\Pi^{+}(\mathbf{D})$ follows similar lines. The only difference is that it does not necessarily follow from $\mathbf{v}^{1} \in \mathcal{K}^{\prime}\left(\mathbf{D}^{1}\right), \mathbf{v}^{2} \in \mathcal{K}^{\prime}\left(\mathbf{D}^{2}\right)$ that $(1-\theta) \mathbf{v}^{1}+\theta \mathbf{v}^{2} \in \mathcal{K}^{\prime}\left[(1-\theta) \mathbf{D}^{1}+\theta \mathbf{D}^{2}\right]$; such a property depends on the definition of the subsets $\mathcal{K}^{\prime}(\mathbf{D})$ of the sets $\mathcal{K}(\mathbf{D})$. If it does hold, then the function $\Pi^{+}(\mathbf{D})$ is convex.

In the case considered in this paper, the subset $\mathcal{K}^{\prime}(\mathbf{D})$ of $\mathcal{K}(\mathbf{D})$ consists of only one velocity field, which depends linearly upon the components of the macroscopic strain rate tensor $\mathbf{D}$; it immediately follows that the above property is satisfied, so that $\Pi^{+}(\mathbf{D})$ is convex. The convexity of the function $\Pi^{+}\left(\bar{D}_{1}, \bar{D}_{2}\right)$ then follows from the fact that the pair $\left(\bar{D}_{1}, \bar{D}_{2}\right)$ is a linear function of $\mathbf{D}$.

\section{F Asymptotes to the curves $\zeta=g(\xi)$ and $\zeta=-g(\xi)$}

The study of the asymptotes to the curves $\zeta=g(\xi)$ and $\zeta=-g(\xi)$ at the points $\xi= \pm \infty$ is based on two main ingredients: (i) the positive homogeneity of degree 1 of the function $\Pi\left(\bar{D}_{1}, \bar{D}_{2}\right)$; (ii) its regularity at the points $\left(\bar{D}_{1}, 0\right), \bar{D}_{1} \neq 0$, arising from the absence of any term proportional to $\left|\bar{D}_{2}\right|$ in the expressions $(21),(22),(23)$ of $\Pi^{(\mathrm{p})}, \Pi^{(\mathrm{s})}, \Pi^{(\mathrm{i})}$.

Let us determine the asymptotic expressions of $g(\xi)$ in the two cases $\xi \rightarrow+\infty$ and $\xi \rightarrow-\infty$ using these properties:

- For $\xi \rightarrow+\infty$ :

$$
\begin{aligned}
g(\xi) & =\Pi(\xi, 1)=\xi \Pi\left(1, \frac{1}{\xi}\right)=\xi\left[\Pi(1,0)+\frac{\partial \Pi}{\partial \bar{D}_{2}}(1,0) \frac{1}{\xi}+O\left(\frac{1}{\xi^{2}}\right)\right] \\
& =\Pi(1,0) \xi+\frac{\partial \Pi}{\partial \bar{D}_{2}}(1,0)+O\left(\frac{1}{\xi}\right) .
\end{aligned}
$$

- For $\xi \rightarrow-\infty$ :

$$
\begin{aligned}
g(\xi) & =\Pi(\xi, 1)=\Pi(-|\xi|, 1)=|\xi| \Pi\left(-1, \frac{1}{|\xi|}\right) \\
& =|\xi|\left[\Pi(-1,0)+\frac{\partial \Pi}{\partial \bar{D}_{2}}(-1,0) \frac{1}{|\xi|}+O\left(\frac{1}{\xi^{2}}\right)\right]=\Pi(-1,0)|\xi|+\frac{\partial \Pi}{\partial \bar{D}_{2}}(-1,0)+O\left(\frac{1}{\xi}\right) \\
& =-\Pi(1,0) \xi-\frac{\partial \Pi}{\partial \bar{D}_{2}}(1,0)+O\left(\frac{1}{\xi}\right)
\end{aligned}
$$


where use has been made of the fact that $\Pi$ is an even function of the pair $\left(\bar{D}_{1}, \bar{D}_{2}\right)$.

It follows from these asymptotic formulae that:

- The curve $\zeta=g(\xi)$ admits straight asymptotes at the points $\xi= \pm \infty$.

- These asymptotes are symmetric with respect to the horizontal $\xi$-axis, since both their slopes and ordinates at the origin are opposite to each other.

- The asymptote to the curve $\zeta=-g(\xi)$ at $\xi=-\infty$ (resp. $\xi=+\infty)$, being the symmetric line of the asymptote to the curve $\zeta=g(\xi)$ at the same point, coincides by what precedes with the asymptote to the curve $\zeta=g(\xi)$ at $\xi=+\infty$ (resp. $\xi=-\infty$ ).

\section{References}

Benzerga, A. A., 2002. Micromechanics of Coalescence in Ductile Fracture. Journal of the Mechanics and Physics of Solids 50, 1331-1362.

Benzerga, A. A., Besson, J., 2001. Plastic potentials for anisotropic porous solids. European Journal of Mechanics 20A, 397-434.

Benzerga, A. A., Besson, J., Batisse, R., Pineau, A., 2002. Synergistic effects of plastic anisotropy and void coalescence on fracture mode in plane strain. Modelling and Simulation in Materials Science and Engineering 10, 73-102.

Benzerga, A. A., Besson, J., Pineau, A., 1999. Coalescence-Controlled Anisotropic Ductile Fracture. Journal of Engineering Materials and Technology 121, 221-229.

Benzerga, A. A., Besson, J., Pineau, A., 2004. Anisotropic ductile fracture. Part II: theory. Acta Materialia 52, 4639-4650.

Benzerga, A. A., Leblond, J.-B., 2010. Ductile fracture by void growth to coalescence. Advances in Applied Mechanics 44, 169-305.

Benzerga, A. A., Leblond, J.-B., 2014. Effective Yield Criterion Accounting for Microvoid Coalescence. Journal of Applied Mechanics 81, 031009.

Cocks, A. C. F., Ashby, M. F., 1982. On creep fracture by void growth. Progress in Materials Science 27, 189-244.

Deshpande, V., Fleck, N., 2000. Isotropic constitutive models for metallic foams. Journal of the Mechanics and Physics of Solids 48, 1253-1283.

Gologanu, M., Leblond, J.-B., Devaux, J., 1993. Approximate models for ductile metals containing non-spherical voids - case of axisymmetric prolate ellipsoidal cavities. Journal of the Mechanics and Physics of Solids 41, 1723-1754.

Gologanu, M., Leblond, J.-B., Devaux, J., 1994. Approximate models for ductile metals containing non-spherical voids-case of axisymmetric oblate ellipsoidal cavities. Journal of Engineering Materials and Technology 116, 290-297.

Gologanu, M., Leblond, J.-B., Perrin, G., Devaux, J., 1997. Recent extensions of Gurson's model for porous ductile metals. In: Suquet, P. (Ed.), Continuum Micromechanics, CISM Lectures Series. Springer, New York, pp. 61-130.

Gurson, A. L., 1977. Continuum Theory of Ductile Rupture by Void Nucleation and Growth: Part I- Yield Criteria and Flow Rules for Porous Ductile Media. Journal of Engineering Materials and Technology 99, 2-15.

Hill, R., 1967. The essential structure of constitutive laws for metal composites and polycrystals. Journal of the Mechanics and Physics of Solids 15, 79-95. 
Keralavarma, S. M., Benzerga, A. A., 2010. A constitutive model for plastically anisotropic solids with non-spherical voids. Journal of the Mechanics and Physics of Solids 58, 874901.

Koplik, J., Needleman, A., 1988. Void growth and coalescence in porous plastic solids. International Journal of Solids and Structures 24, 835-853.

Leblond, J.-B., Mottet, G., 2008. A theoretical approach of strain localization within thin planar bands in porous ductile materials. Comptes Rendus Mécanique 336, 176-189.

Madou, K., Leblond, J.-B., 2012a. A Gurson-type criterion for porous ductile solids containing arbitrary ellipsoidal voids - I: Limit-analysis of some representative cell. Journal of the Mechanics and Physics of Solids 60, 1020-1036.

Madou, K., Leblond, J.-B., 2012b. A Gurson-type criterion for porous ductile solids containing arbitrary ellipsoidal voids - II: Determination of yield criterion parameters. Journal of the Mechanics and Physics of Solids 60, 1037-1058.

Madou, K., Leblond, J.-B., 2013. Numerical studies of porous ductile materials containing arbitrary ellipsoidal voids - I: Yield surfaces of representative cells. European Journal of Mechanics 42, 480-489.

Madou, K., Leblond, J.-B., Morin, L., 2013. Numerical studies of porous ductile materials containing arbitrary ellipsoidal voids - II: Evolution of the length and orientation of the void axes. European Journal of Mechanics 42, 490-507.

Mandel, J., 1964. Contribution théorique à l'étude de l'écrouissage et des lois d'écoulement plastique. In: $11^{\text {th }}$ International Congress on Applied Mechanics. Springer, Berlin, pp. $502-509$.

McClintock, F. A., 1968. A criterion for ductile fracture by the growth of holes. Journal of Applied Mechanics 35, 363-371.

Monchiet, V., Cazacu, O., Charkaluk, E., Kondo, D., 2008. Macroscopic yield criteria for plastic anisotropic materials containing spheroidal voids. International Journal of Plasticity 24, 1158-1189.

Morin, L., Leblond, J.-B., Benzerga, A. A., 2015a. Coalescence of voids by internal necking: Theoretical estimates and numerical results. Journal of the Mechanics and Physics of Solids $75,140-158$.

Morin, L., Leblond, J.-B., Kondo, D., 2015b. A Gurson-type criterion for plastically anisotropic solids containing arbitrary ellipsoidal voids. International Journal of Solids and Structures In Press.

Pardoen, T., Hutchinson, J. W., 2000. An extended model for void growth and coalescence. Journal of the Mechanics and Physics of Solids 48, 2467-2512.

Perrin, G., 1992. Contribution à l'étude théorique et numérique de la rupture ductile des métaux. Ph.D. thesis, Ecole Polytechnique.

Ponte Castaneda, P., Suquet, P., 1997. Nonlinear composites. Advances in Applied Mechanics 34, 171-302.

Prager, W., Hodge, P. G., 1951. Theory of Perfectly Plastic Solids. Applied Mathematics Series. John Wiley \& Sons.

Rice, J. R., Tracey, D. M., 1969. On the enlargement of voids in triaxial stress fields. Journal of the Mechanics and Physics of Solids 17, 201-217.

Rudnicki, J. W., Rice, J. R., 1975. Conditions for the localization of deformation in pressure-sensitive dilatant materials. Journal of the Mechanics and Physics of Solids 23, 371-394.

Suquet, P., 1983. Limit analysis and homogenization. Comptes Rendus Académie des 
Sciences Paris 296, 1355-1358.

Tekoglu, C., Leblond, J.-B., Pardoen, T., 2012. A criterion for the onset of void coalescence under combined tension and shear. Journal of the Mechanics and Physics of Solids 60, $1363-1381$.

Thomason, P. F., 1985. Three-dimensional models for the plastic limit-loads at incipient failure of the intervoid matrix in ductile porous solids. Acta Metallurgica 33, 1079-1085.

Torki, M. E., Benzerga, A. A., Leblond, J.-B., 2015. On Void Coalescence Under Combined Tension and Shear. Journal of Applied Mechanics 82, 071005.

Tvergaard, V., 1981. Influence of voids on shear band instabilities under plane strain conditions. International Journal of Fracture 17, 389-407.

Tvergaard, V., Needleman, A., 1984. Analysis of the cup-cone fracture in a round tensile bar. Acta Metallurgica 32, 157-169.

Yamamoto, H., 1978. Conditions for shear localization in the ductile fracture of voidcontaining materials. International Journal of Fracture 14, 347-365.

Zhang, Z. L., Niemi, E., 1994. Analyzing ductile fracture using dual dilational constitutive equations. Fat. Frac. Eng. Mater. Structures 17, 695-707. 